\title{
Deep Learning Based Hybrid Models for Prediction of COVID- 19 using Chest X-Ray
}

This paper was downloaded from TechRxiv (https://www.techrxiv.org).

\section{LICENSE}

CC BY 4.0

SUBMISSION DATE / POSTED DATE

$21-08-2020$ / 21-08-2020

\section{CITATION}

R, Shree Charran; Dubey, Rahul Kumar (2020): Deep Learning Based Hybrid Models for Prediction of COVID19 using Chest X-Ray. TechRxiv. Preprint. https://doi.org/10.36227/techrxiv.12839204.v1

DOI

10.36227/techrxiv.12839204.v1 


\title{
Deep Learning Based Hybrid Models for Prediction of COVID-19 using Chest X-Ray
}

\author{
Shree Charran R and, Rahul Kumar Dubey
}

\begin{abstract}
COVID-19 has ended up being the greatest pandemic to come to pass for on humanity in the last century. It has influenced all parts of present day life. The best way to confine its spread is the early and exact finding of infected patients. Clinical imaging strategies like Chest X-ray imaging helps specialists to assess the degree of spread of infection. In any case, the way that COVID-19 side effects imitate those of conventional Pneumonia brings few issues utilizing of Chest $\mathrm{X}$ rays for its prediction accurately. In this investigation, we attempt to assemble 4 ways to deal with characterize between COVID-19 Pneumonia, NON-COVID-19 Pneumonia, and an Healthy- Normal Chest X-Ray images. Considering the low accessibility of genuine named Chest X-Ray images, we incorporated combinations of pre-trained models and data augmentation methods to improve the quality of predictions. Our best model has achieved an accuracy of $99.5216 \%$. More importantly, the hybrid did not predict a False Negative Normal (i.e. infected case predicted as normal) making it the most attractive feature of the study.
\end{abstract}

Index Terms - COVID-19, Pneumonia, Chest X-Ray, Model Ensemble

\section{INTRODUCTION}

$\mathrm{T}$ THE COVID-19 coronavirus pandemic is the leading public health epidemic of the decade and the greatest threat facing humanity since World War Two. The virus has spread to every continent except the Antarctica since its arrival in Asia in late 2019. Across Africa the Americas, and in Europe, cases are rising daily. Currently, COVID-19 testing kits are hard to get - there's just not enough of them and they can't be manufactured fast enough and that's creating great chaos. This has resulted to the selling of bogus COVID-19 test kits and other threats to the public. The limited COVID-19 test kits results in an urgent need to focus on other methods for diagnosis. As COVID19 attacks the epithelial cells which line our respiratory tract, we can use X-rays to examine the health of the lungs of a patient. Furthermore, provided that all major hospitals have access to X-ray imaging equipment, without the special test sets, X-rays could be used to monitor for COVID-19.

The indications of COVID-19 pneumonia might be like different sorts of viral pneumonia. Along these lines, it tends to be hard to determine what's causing your condition without being tried for COVID-19 or other respiratory diseases. Exploration is in progress to decide how COVID19 pneumonia contrasts from different sorts of pneumonia. Data from these investigations can conceivably help in finding and in facilitating our comprehension of how SARS$\mathrm{CoV}-2$ influences the lungs. CT \& Xray Imaging \& laboratory tests to compare the clinical features to differentiate COVID-19 pneumonia to different sorts of pneumonia. Scientists have found that individuals with COVID-19 pneumonia were bound to have: 1) pneumonia that influences the two lungs rather than only one 2)lungs that had a trademark "ground-glass" appearance by means of CT check 3)abnormalities in some research tests, especially those evaluating liver capacity. This clearly indicates that there is considerable room for use of $\mathrm{AI}$ in differentiating COVID-19 to viral pneumonia.

The Computer Vision groups across the globe has made a huge efforts over the last decade and made many State of the Art models open to the public. These State-of-the-art models are conditioned on various data types and are fine-tuned for certain typical tasks and purposes. For this analysis we expect to use various state of the art capabilities and variations of the same to create a powerful prediction model to classify between COVID-19, Non- COVID Pneumonia, and Normal.

\section{Related Work}

Kassani et al. [16] introduced a feature extractor-based multi method ensemble approach for computer-aided analysis of COVID-19 pneumonia. 6 Machine learning algorithms were trained on the features extracted by by State of the art CNNs to find the best combination of features and learners. Considering the high visual complexity of image data, proper deep feature extraction is considered as a critical step in developing deep CNN models. he experimental results on the chest $\mathrm{X}$-ray datasets showed that the features extracted by Dense-Net-121 and finally trained using the Bagging tree classifier generates best predictions with $99.00 \%$ classification accuracy.

Wang and Wong[21] introduced COVID-Net, to detect COVID-19 from X-ray images of the chest. The COVIDNet architecture was designed from a mixture of $1 \mathrm{x} 1$ convolutions, depth-wise convolution, and residual modules to allow for deeper system design and prevent the issue of gradient disappearing. The dataset given was a mix of COVID chest X-ray dataset provided by [17], and Kaggle chest X-ray images dataset [22] for multi-class classification of multi-class classification of normal vs bacterial vs COVID-19 infection dataset. The obtained accuracy of this study was 83.5 per cent.

Khan et al.[23] proposed CoroNet, to automatically detect COVID-19 from chest X-ray images. Coronet was built using the Xception architecture with ImageNet weights. CoroNet achieved an overall accuracy of $89 \%$, precision of $93 \%$ and recall of $98.2 \%$ for 4 -class cases they being COVID-19, Viral and bacterial Pneumonia and Healthy. The same model achieved $95 \%$ accuracy for 3class classification i.e COVID-19, Pneumonia and Healthy. Chouhan et al.[26] proposed a deep learning approach to classify pneumonia from chest X-rays using State of the art 
pre-trained models. They tested the performances of State of the art pre-trained models like AlexNet, DenseNet, Inception V3 etc to extract features. The extracted features were passed through individual classifiers and the predictions of individual architectures were obtained. An overall ensemble of all five pretrained models was observed to outperform all other models.

Rajaraman et al.[27] studied and found that performing Reiterative pruning and selecting the best pruned model improved the prediction accuracies and further helped minimize parameter numbers as redundant parameters which do not help improve the prediction performance are eliminated. Further they were able to better the performance by use of ensembles of pruned models. Awarding weights based on their predictions, the authors observed that the weighted averaging ensemble of the pruned models outperformed the other ensemble methods. Overall it was identified that combinations of iterative pruning of models and ensembles of models helped reduce prediction variance, model complexity.

In this paper, we evaluate four major ensemble hybrids from pre trained State of the art models to build an better ensemble which neutralizes deficiencies of individual architectures.

\section{Modelling}

\subsection{Feature Ensembles:}

The baseline models are initialized with ImageNet weights are used to extract the image features. To extract image features, final softmax layers are removed. The combined dimensions of these multi models are reduced using a standard model technique PCA. The PCA components are selected to explain $90 \%$ of the total variance. Which is finally passed through a dense 256 layer and a softmax for final predictions. The architecture of feature ensemble for the baseline model depicted in Figure 1.

Fig .1: Feature ensemble for the baseline model

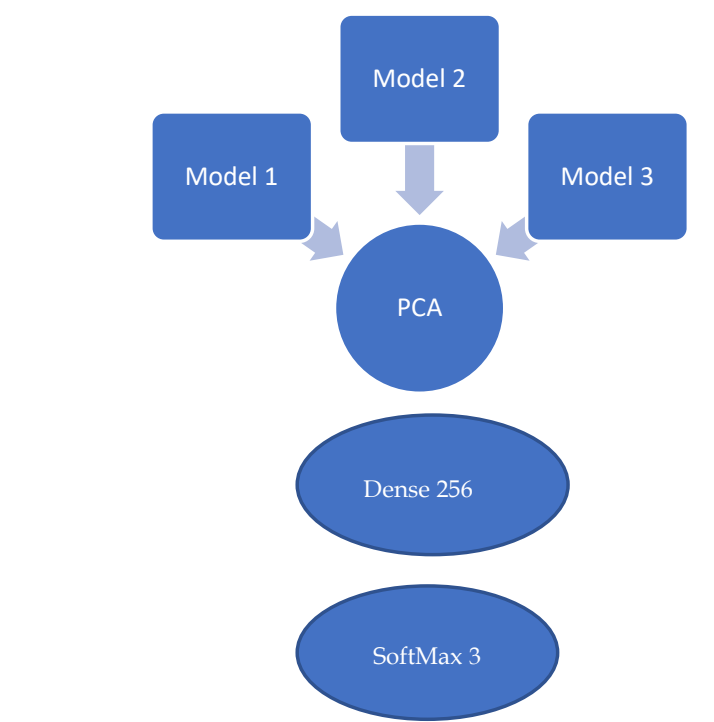

Fig : Feature ensemble for the baseline model

\subsection{Majority Voting:}

This is a naïve but effective approach .The main Baseline models are individually assessed on the dataset and individual predictions are made. The prediction vector constitutes the individual predictions of the models. Majority voting system is used to make a final prediction from the prediction vector. Further in case of a voting clash, the best single classifier vote is doubled. Figure 2 depicts the above method.

\subsection{Feature Classification:}

The Standard procedure is to extract the image features after pre-processing, and the extracted features are put into the classifier for training. Finally, the model after training is tested. For the process of feature extraction, the baseline model is used for extracting features in this method. For the process of classification, three classification models are selected to classify the extracted features in this paper. The selected classification models are SVM[20], Bagging Classifier[19] and AdaBoost[18] to have variety.

\subsection{Layer Modification:}

The baseline models are initialized with ImageNet weights was trained on the augmented dataset training set to optimize network weights. During training, early stopping was implemented using 3 epochs measure. The final softmax layer, mapping to 3 output classes, was replaced with 2 dense layers, $50 \%$ dropout layer, and softmax layer mapping to the X-ray labels. These layers were introduced to maximize baseline model classification accuracy during the transfer learning process. To extract model features, the top dense layer was removed, along with the dropout layer, and the output of the previous dense layer was used as a feature vector resulting in a 512-length labelled feature vector per image. The architecture of layer modification for baseline model depicted in Figure 3.

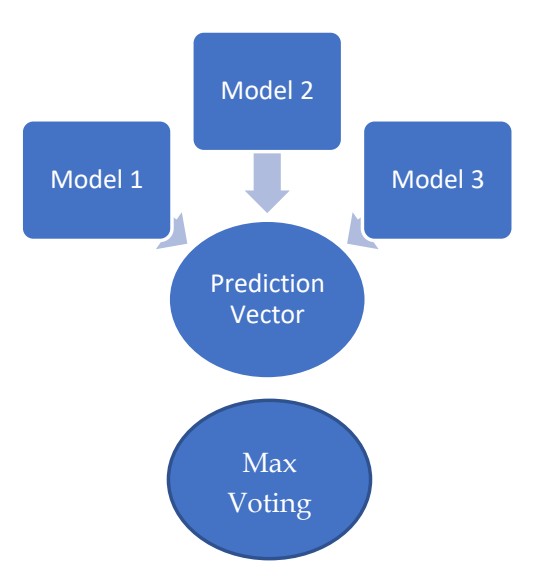

Fig. 2: Max voting ensemble from the baseline model 


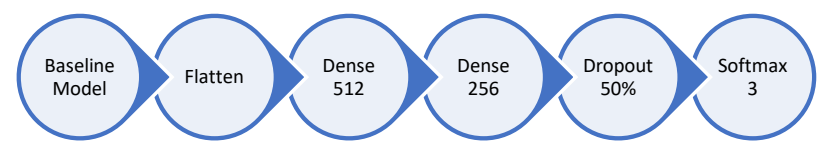

Fig. 3: Layer modification for baseline model

\section{EXPERIMENTAL SETUP}

\subsection{Baseline Models:}

In this section, we explain in brief about the selected pretrained models which we will use as baseline models and for experiments explained in section 3 .

\subsubsection{VGG-16 [1]:}

VGG16 is a convolution neural net (CNN ) network which was utilized to win Imagenet competition in 2014. Most remarkable thing about VGG16 is that as opposed to having countless hyper-parameter they concentrated on having convolution layers of $3 \times 3$ channel with a step 1 and consistently utilized same padding space and maxpool layer of $2 \times 2$ channel of stride 2 . It follows this game plan of convolution and max pool layers reliably all through the entire network. At the last it has 2 fully associated layers with a softmax for final output. The 16 in VGG16 refers to it has 16 layers that have the weights. This system is a truly huge system and it has around 138 million parameters.

\subsubsection{RESNET 50 [2]:}

ResNet, short for Residual Networks is a neural network. This model was the winner of ImageNet challenge in 2015. The forward leap with ResNet was it allowed us to train extremely deep neural networks with 150+layers successfully. Prior to ResNet training very deep neural networks was difficult due to the problem of vanishing gradients.There are numerous variations of ResNet for example same idea yet with an different number of layers. We have ResNet-50, ResNet-101, ResNet-110, ResNet-152 and so forth. The name ResNet followed by a two or more digit number basically suggests the ResNet design with a specific number of neural layers. ResNet-50 is one of the most compact and vibrant networks.

The architecture of ResNet50 has 4 stages. The network can take the input image having height, width as multiples of 32 and 3 as channel width. Every ResNet architecture performs the initial convolution and maxpooling using $7 \times 7$ and $3 \times 3$ kernel sizes respectively. Afterward, Stage 1 of the network starts and it has 3 Residual blocks containing 3 layers each. The size of kernels used to perform the convolution operation in all 3 layers of the block of stage 1 are 64,64 and 128 respectively. the convolution operation in the Residual Block is performed with stride 2 , hence, the size of input will be reduced to half in terms of height and width but the channel width will be doubled.

As we progress from one stage to another, the channel width is doubled and the size of the input is reduced to half. For deeper networks like ResNet50, ResNet152, etc, bottleneck design is used. For each residual function F, 3 layers are stacked one over the other. The three layers are $1 \times 1,3 \times 3,1 \times 1$ convolutions. The $1 \times 1$ convolution layers are responsible for reducing and then restoring the dimensions. The $3 \times 3$ layer is left as a bottleneck with smaller input/output dimensions. Finally, the network has an Average Pooling layer followed by a fully connected layer having 1000 neurons.

\subsubsection{INCEPTION V3 [3]:}

Inception $\mathrm{v} 3, \quad$ is developed from GoogLeNet/Inception-v1. Inception V1 was the best in class design at Imagenet Competition 2014. It has created the record lowest error rate at ImageNet dataset yet there are a few focuses on which improvement was made to improve the accuracy and decrease the complexity of the model.

Inception $\mathrm{V} 3$ has the following changes :

- Utilization of RMSprop optimizer.

- Batch Normalization in the fully connected layer of Auxiliary classifier.

- Utilization of $7 \times 7$ factorized Convolution

- Label Smoothing Regularization: $t$ is a method to regularize the classifier by estimating the effect of label-dropout during training. It prevents the classifier to predict too confidently a class. The addition of label smoothing gives $0.2 \%$ improvement from the error rate.

We chose these architectures because they are well established and have shown good performance when adapted to a variety of medical image classification scenarios [4-6]. Table 1 highlights the same.

TABLE 1

KEY POINTS OF THE BASELINE MODELS

\begin{tabular}{|l|l|l|}
\hline Model & Connection Type & Parameters \\
\hline VGG-16 & Fixed-kernel & $138 \mathrm{M}$ \\
\hline ResNet-50 & Shortcut & $23 \mathrm{M}$ \\
\hline Inception V3 & Wider- Parallel & $24 \mathrm{M}$ \\
\hline
\end{tabular}

\subsection{Dataset:}

The chest X-ray images are obtained from free-publicly available $\mathrm{X}$-ray datasets:

i) Kaggle Pneumonia dataset (1583 normal xray +4273 pneumonia $x$-ray)[7]. 
ii) Kaggle Covid-chest Dataset (150 Covid-19) [9].

iii) GitHub UCSD-AI4H/COVID-CT (288 Covid19 x-ray)[10].

iv) SIIM.org (60 Covid-19 x-ray)[11].

v) University of Montreal (684 Covid-19 x-ray) [8].

From Figure 4 it can be noticed that there are limited images available for the COVID-19 case. We will be incorporating a data augmentation strategy to partial rectify this skew in data. Also, Figure 5, Figure 6 and Figure 7 shows samples from the dataset depicting the 3 variants of sample.

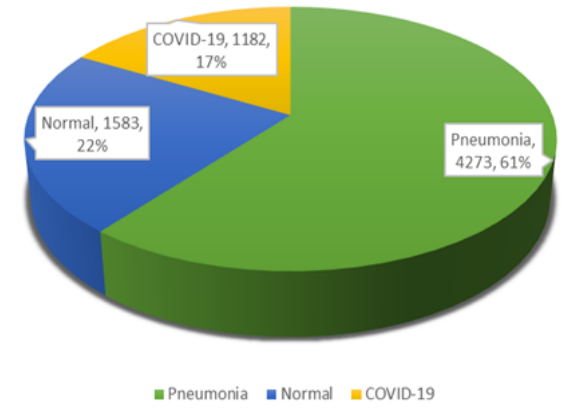

Fig. 4: Distribution of chest $\mathrm{X}$-ray types used

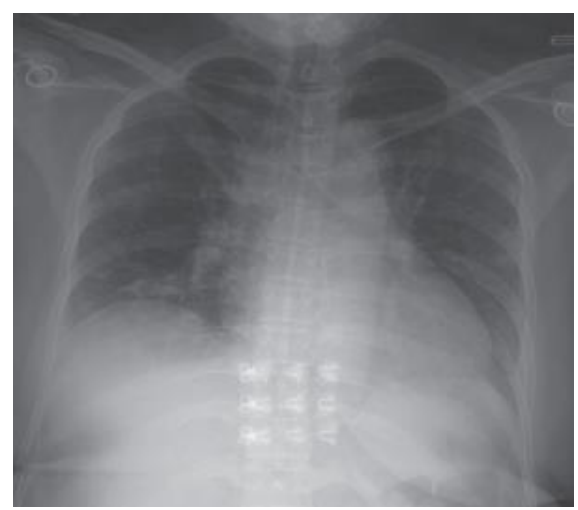

Fig. 5 : Covid-19 positive chest X-ray

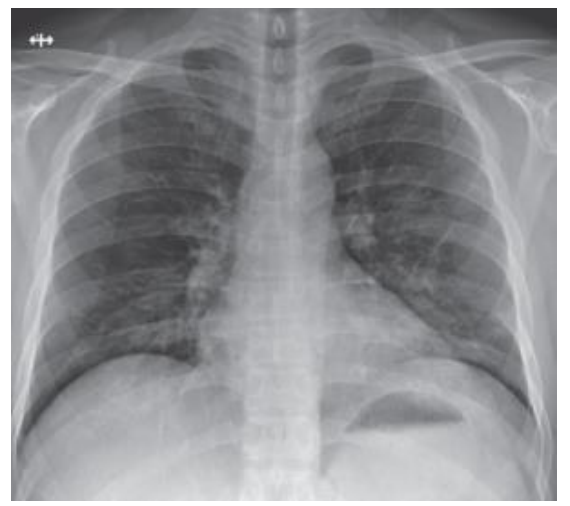

Fig. 6: Pneumonia (positive) chest $\mathrm{x}$-ray

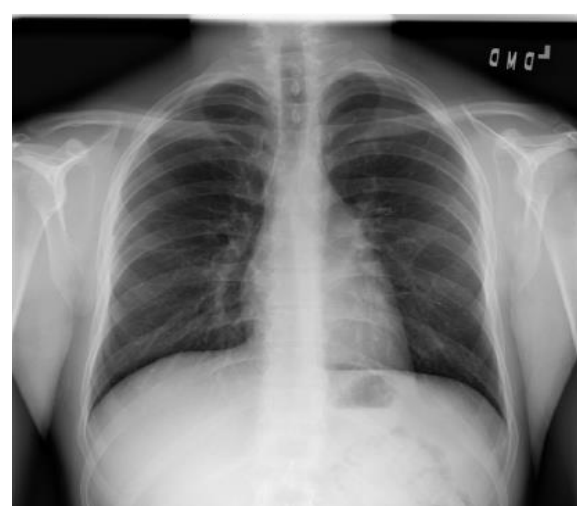

Fig. 7: Healthy chest $x$-ray

\subsection{Data Augmentation:}

It must be noted that X-ray images are usually of the resolution 1024 pixels x 1024 pixels and are single-channel images and not RGB. Firstly Cropping of the images should not be performed on Xray images to ensure abnormalities within the images is not missed. Therefore we find the augmentation strategies a good option:

a) Flip: We perform separate horizontal flip and vertical flip for each image in the dataset.

b) Rotation: Rotation of images is done using the following transformation,

$$
A=\left(\begin{array}{cc}
\cos \theta & -\sin \theta \\
\sin \theta & \cos \theta
\end{array}\right)
$$

where $\theta$ is between 10 and 80 degrees, is applied

c) Gaussian Noise: An array, A, is generated where each element in the array is a sample from a gaussian distribution with $\mu=0$ and with $\sigma 2$ in the range of $[0.1,0.9]$. For each image $\mathrm{X}$ in the dataset, we obtain a noisy image, $\mathrm{X}^{\prime}=\mathrm{X}+\mathrm{A}$.

d) Jitter: For each image in the dataset, we add a small amount of contrast (+/- 1-5 intensity values).

e) Power: For each Image in the dataset we take it to power. The power, $p$, is given by:

$$
\mathbf{p}=\mathbf{n} \times \mathbf{r}+\mathbf{1}
$$

where $\mathrm{n}$ is a number taken from a Gaussian distribution with mean 0 and variance 1 while $r$ is a number $<1$. Then, the augmented image, $X_{a}$, is given by,

$$
X_{\mathrm{a}}=\operatorname{sign}(X) *\left(|X|^{p}\right)
$$

The sign and power are each taken elementwise. 
f) Gaussian Blur: A function defined by the variance between 0.1 and $0.9 .(r=3, \sigma$.$) is applied to blur the$ images

g) Shearing : For each image in the dataset, the following transformation is done,

$$
A=\left(\begin{array}{ll}
1 & s \\
0 & 1
\end{array}\right)
$$

$\mathrm{S}$ is the amount that image is to be sheared, and it is in the range of $[0.1,0.35]$.

\subsection{Other Preprocessing:}

The images vary in quality and dimension, ranging from $1215 \times 759$ pixels to $1024 \times 1024$ pixels due to multiple sources ; therefore, we will bring them to the size of $778 \mathrm{x}$ 778 pixels to obtain a constant dimension for various images used as input images.

\subsection{Evaluation Metrics:}

Standard metrics for classification derived from the confusion matrix are used for evaluation. The Table 2 provides the Confusion Matrix for a 2 Class case.

TABLE 2

CONFUSION MATRIX

\begin{tabular}{|c|c|c|c|}
\hline \multirow{4}{*}{ 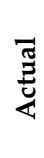 } & \multicolumn{3}{|c|}{ Predicted } \\
\hline & & Negative & Positive \\
\hline & Negative & True Negative & False Positive \\
\hline & Positive & False Negative & True Positive \\
\hline
\end{tabular}

\subsubsection{ACCURACY}

Classification accuracy is a naïve metric . It is the number of correct predictions made divided by the total number of predictions made. Accuracy in confusion metric terms is given by:

Accuracy $=$

True positive + True negative

$\overline{\text { True positive }+ \text { True negative }+ \text { False positive }+ \text { False negative }}$

\subsubsection{PreCISION}

Precision can be thought of as a measure of a classifiers exactness. A low precision indicates a large number of False Positives. Precision in confusion metric terms is given by:

$$
\text { Precision }=\frac{\text { True positive }}{\text { True positive }+ \text { False positive }}
$$

\subsubsection{RECALL:}

Recall calculates how many of the Actual

Positives our model capture through labeling it as Positive (True Positive). Recall shall be the model metric we use to select our best model when there is a high cost associated with False Negative. Thus in Covid patient detection. If a Covid patient (Actual Positive) goes through the test and predicted as not sick (Predicted Negative). The cost associated with False Negative will be extremely high if the sickness is contagious.. The recall in confusion metric terms is given by:

$$
\text { Recall }=\frac{\text { True positive }}{\text { True positive }+ \text { False negative }}
$$

\subsubsection{F-1 SCORE:}

F1 Score is a good measure to use if we need to seek a balance between Precision and Recall and there is an uneven class distribution (COVID samples).

F1-Score in confusion metric terms is given by:

$$
\text { F1-Score }=\frac{2 * \text { Recall } * \text { Precision }}{\text { Recall }+ \text { Precision }}
$$

\subsection{Experimental Details:}

The primary goal of our transferring learning approach was to correctly diagnose COVID vs pneumonia among normal chest X-ray images. For this, we prepared all of the models and trained them separately. For training, we used Adam optimizer and the cross-entropy loss function. The learning rate is started from the value of 0.001 and is reduced by 1 after every 5 epochs. The early stopping function takes care of the epoch number. The Training images after augmentation processes and duplication removal was 211142 and $10 \%$ of this was held for testing.

\section{RESUlts \& DisCusSION:}

We have conducted the experiments using the methodology discussed in section 3. The details are as below.

- Baseline Study:

The pre-trained models have experimented as individuals without any alterations to get a benchmark.

- Hybrid 1: The feature ensemble model, features extracted from VGG-16, ResNet-50, Inception V3 are reduced with PCA and passed through a dense layer and SoftMax.

- Hybrid 2: The predictions of VGG-16, ResNet-50, and Inception V3 are passed through a max voting layer for final output.

- Hybrid 3: Modified Architecture of the 3 models are individually tested

- Hybrid 4: features extracted from VGG-16, ResNet50 , Inception V3 are passed separately through the classifiers to find best settings 
TABLE 3

SUMMARY OF RESULTS

\begin{tabular}{|c|l|l|c|c|c|}
\hline \multirow{3}{*}{ Type } & Models & $\begin{array}{l}\text { Accuracy } \\
(\mathbf{\%})\end{array}$ & $\begin{array}{l}\text { Precision } \\
(\mathbf{\%})\end{array}$ & $\begin{array}{l}\text { Recall } \\
(\mathbf{\%})\end{array}$ & F1- Score \\
\hline \multirow{5}{*}{ Baseline } & VGG-16 & 90.19 & 94.12 & 94.16 & 94.14 \\
\cline { 2 - 6 } & ResNet-50 & 87.28 & 92.12 & 91.18 & 91.65 \\
\cline { 2 - 6 } & Inception V3 & 82.22 & 85.12 & 85.24 & 85.18 \\
\hline \multirow{3}{*}{ Hybrid 1 } & Feature Ensembles & 91.76 & 95.88 & 95.60 & 95.74 \\
\hline \multirow{3}{*}{ Hybrid 2 } & Majority Voting & 92.19 & 96.33 & 96.06 & 96.19 \\
\hline & VGG-16 Modified & $\mathbf{9 9 . 5 2}$ & $\mathbf{9 9 . 7 7}$ & $\mathbf{9 7 . 9 3}$ & $\mathbf{9 8 . 8 4}$ \\
\cline { 2 - 6 } & ResNet-50 Modified & 97.75 & 95.80 & 94.83 & 95.31 \\
\cline { 2 - 6 } & Inception V3 Modified & 92.09 & 95.33 & 95.47 & 95.40 \\
\hline & VGG-16 SVM & 91.19 & 94.12 & 93.15 & 93.63 \\
\cline { 2 - 6 } & VGG-16 Bagging & 90.19 & 92.22 & 92.16 & 92.19 \\
\cline { 2 - 6 } & VGG-16 AdaBoost & 90.19 & 89.16 & 90.10 & 89.63 \\
\cline { 2 - 6 } & ResNet-50 SVM & 96.11 & 89.12 & 89.12 & 89.12 \\
\cline { 2 - 6 } & ResNet-50 Bagging & 95.12 & 95.12 & 95.07 & 95.09 \\
\cline { 2 - 6 } & ResNet-50 AdaBoost & 90.18 & 88.12 & 88.12 & 88.12 \\
\cline { 2 - 6 } & Inception V3 SVM & 84.29 & 85.12 & 85.20 & 85.16 \\
\cline { 2 - 6 } & Inception V3 Bagging & $\mathbf{9 9 . 3 6}$ & $\mathbf{9 9 . 3 6}$ & $\mathbf{9 9 . 1 2}$ & $\mathbf{9 9 . 2 4}$ \\
\cline { 2 - 6 } & Inception V3 AdaBoost & 80.12 & 85.00 & 86.12 & 85.56 \\
\hline
\end{tabular}

The Accuracy, Precision, Recall and F1 score are reported in Table 3. From Figure 8 and Table 3 It can be observed that the base VGG-16 outperforms the other 2 base models. Whereas the Inception V3 performs very poorly in comparison with an accuracy of $82.22 \%$.

Hybrid 1: The combined feature extraction and PCA help exploit the feature selection process of multiple models and develop a union feature set from

them. This enhanced feature selection outperforms the individual feature selection capacity of the models.

In Hybrid 2: The combined prediction power of multiple models seems to easily outperform the baseline models. This can be expected. Different models are pretrained on different images. This combination helps exploit the power of individuals. Figure 9 compares both Hybrid 1 and Hybrid 2 . It can be seen both have better performance than the individual models. This highlights the advantage and the power of ensembling.

Hybrid 3: modified architecture has significantly improved the individual score of the models by an average of $9.8 \%$ (Figure 10). This is because of the extended architecture of the model which further fine-tunes the vector space. It also is observed that the F-1 score of the Inception V3 model beats the ResNet-50 because of a better recall. Figure 10 depict the performance of Hybrid 3.

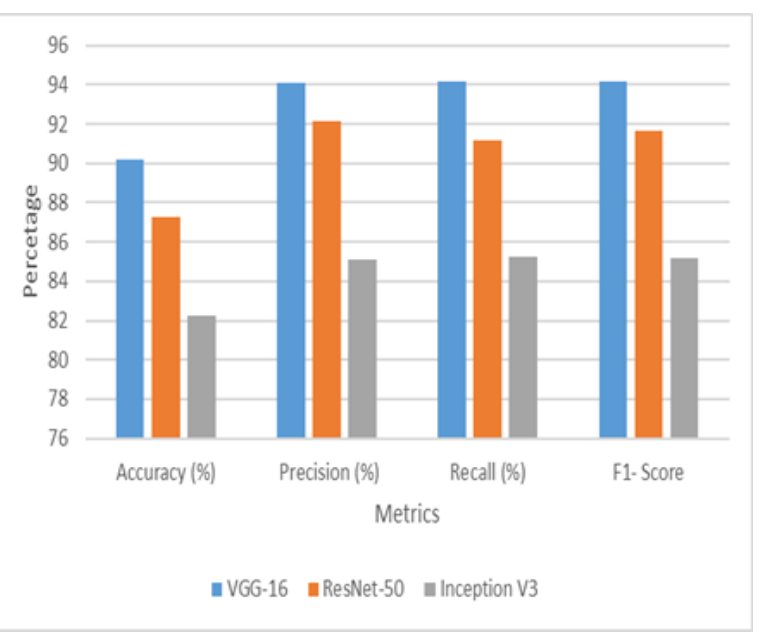

Fig. 8: Results of baseline models

In Hybrid 4: Analyzing Table 3 it can be observed for hybrid 4 the topmost result was obtained by bagging classifier. Figure 11 depict the performance of Hybrid 4. The Bagging classifier performs best for all 3 models. The Inception V3- Bagging variant. 


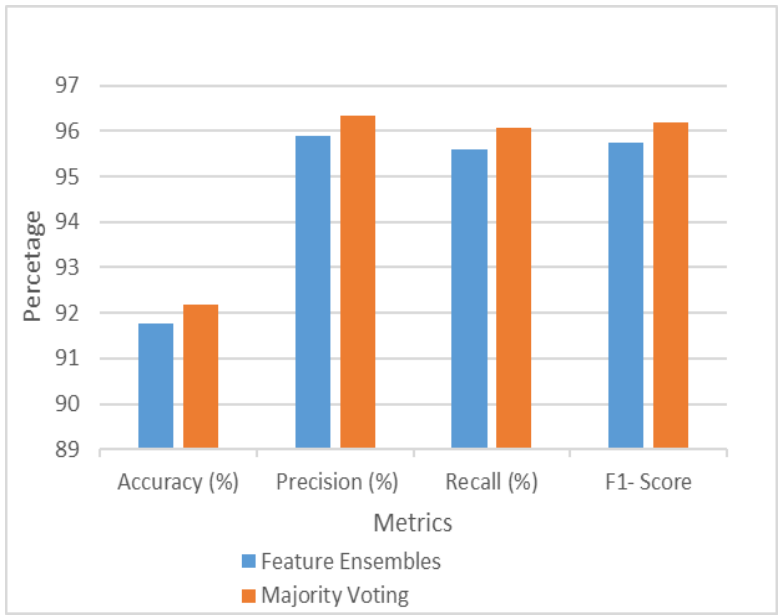

Fig. 9: results of hybrid 1(feature ensemble) \& hybrid 2 (max voting)

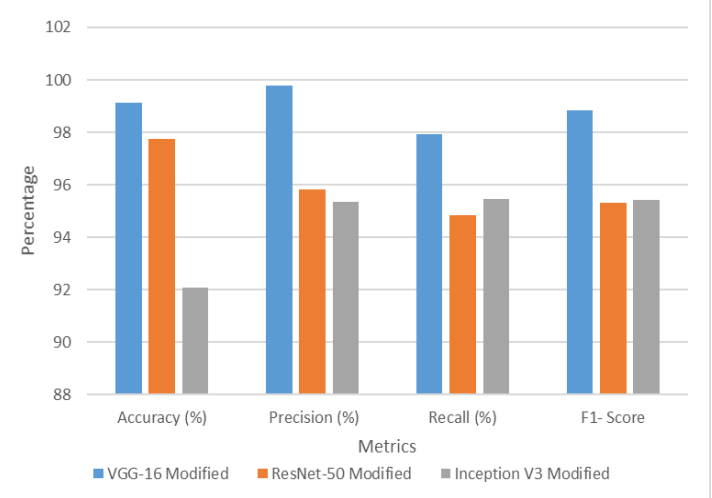

Fig. 10: Results of hybrid 3

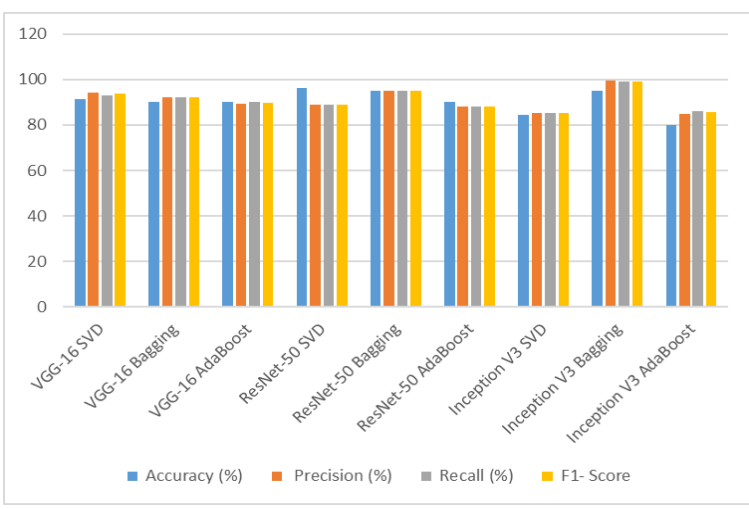

Fig. 11: Results of hybrid 4

Performs outstandingly with $99.36 \%$ accuracy (135/21114 misclassified). Overall Observations being the best performer is the modified VGG-16 with an accuracy of $99.52 \%$ followed by the Inception V3 - Bagging model. Table 4 gives the confusion matrix of the multi-class prediction of the Modified VGG-16 model. It can be seen in a test set of 21114 images only 101 were misclassified. This brings the accuracy to $99.52 \%$ which is the best recorded among many research papers on the topic. Another breakthrough is the fact that there were ZERO cases where the infection was recorded as normal (False Negative Normal). Figure 11 compares results of hybrid4.The Confusion Matrix of the best model is shown in Table 4. It shows that No True cases come out as normal. Making this the highlight of the Modified VGG-16 model

TABLE 4 CONFUSION MATRIX MODIFIED VGG-16

\begin{tabular}{|c|c|c|c|c|}
\hline \multirow{5}{*}{ 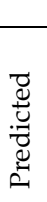 } & & \multicolumn{3}{|c|}{ TRUE } \\
\hline & & Covid-19 & Pneumonia & Normal \\
\hline & Covid-19 & 3523 & 25 & 0 \\
\hline & Pneumonia & 76 & 12765 & 0 \\
\hline & Normal & 0 & 0 & 4725 \\
\hline
\end{tabular}

\section{Conclusions:}

In this paper, our goal is to explore major ensemble approaches to classify COVID-19 and pneumonia from chest $\mathrm{X}$-ray images using various available transfer learning methods. In this paper, we adopted the pre-trained architectures, Inception V3, VGG-16, and ResNet18 trained on the ImageNet dataset, to extract features. The modified VGG-16 and the Inception v3 + Bagging achieved an accuracy of $99.52 \%$ and $99.36 \%$ accuracy respectively. We observed that performance could be improved further, by increasing dataset size, using a data augmentation approach, and using hand-crafted features, in the future. Our findings support the notion that deep learning - AI approaches can be used to improve and ease the diagnostic process and improve disease management.

While contamination analysis are usually done and managed by a single specialist, taking into account the chance of mistake, deep learning strategies can be viewed as a two-way confirmation. For this situation, deep network gives an analysis dependent on chest X-ray pictures, which would then be able to be affirmed by the going to doctor, definitely limiting both human and PC mistake. Our outcomes propose that profound learning strategies can be utilized to improve analysis comparative with conventional techniques, which may improve the nature of treatment. When contrasted and the past cutting edge strategies, our methodology can viably identify the inflammatory region in chest X-ray pictures .

\section{REFERENCES}

[1] Karen Simonyan and Andrew Zisserman, "Very deep convolutional networks for large-scale image recognition," in International Conference on Learning Representations, 2015.

[2] He, Kaiming, et al. "Deep residual learning for image recognition." Proceedings of the IEEE conference on computer vision and pattern recognition. 2016.

[3] C. Szegedy et al., "Going deeper with convolutions", Proc. IEEE Conf. Comput. Vis. Pattern Recognit., pp. 1-9, Jun. 2015.

[4] N. Tajbakhsh et al., "Convolutional neural networks for medical image analysis: Full training or fine-tuning?" IEEE Trans. Med. Imag., vol. 35, no. 5, pp. 1299-1312, May 2016. 
[5] J. Margeta, A. Criminisi, R. C. Lozoya, D. Lee, and N. Ayache, "Finetuned convolutional neural nets for cardiac MRI acquisition plane recognition," Comput. Method Biomech. Biomed. Eng. Imag. Vis., 2016. DOI: 10.1080/21681163.2015.1061448.

[6] S. Choi, "X-ray image body part clustering using deep convolutional neural Network: SNUMedinfo at imageCLEF 2015 medical clustering task," in Proc. Workshop CLEF 2015 Working Notes, 2015.

[7] G, Shih, et al., "Augmenting the National Institutes of Health Chest Radiograph Dataset with Expert Annotations of Possible Pneumonia", Radiol Artif Intell., vol. 1, no. 1, pp. 1-5, 2019.

[8] J. P. Cohen, P. Morrison, and L. Dao, COVID-19 image data collection, 2020, [Online] Available: https://arxiv.org/abs/2003.11597.

[9] Kaggle.Available:https://www.kaggle.com/andrewmvd/convid19xrays

[10] GitHub https://github.com/ieee8023/covid-chestxray-dataset from April 16, 2020-May 5, 2020

[11] SIIM.org https://SIIM.org/covid-chestxray-dataset

[12]D. J. Mollura, P. M. Lungren, Radiology in Global Health, Strategies, Implementation, and Applications, Springer, 2019. Crossref, Google Scholar

[13] E. J. Hwang, J. G. Nam, W. H. Lim, S. J. Park, Y. S. Jeong, J. H. Kang, E. K. Hong, T. M. Kim, J. M. Goo, S. Park, K. H. Kim, C. M. Park, "Deep Learning for Chest Radiograph Diagnosis in the Emergency Department," Radiology, vol. 293, no. 3, 2019. Link, Google Scholar

[14] M. Annarumma, S. J. Withey, R. J. Bakewell, E. Pesce, V. Goh, G. Montana, "Automated Triaging of Adult Chest Radiographs with Deep Artificial Neural Networks," Radiology, vol. 291, no. 1, 2019. Link, Google Scholar

[15]K. Murphy, S. S. Habib, S. M. A. Zaidi, S. Khowaja, A. Khan, J. Melendez, E. T. Scholten, F. Amad, S. Schalekamp, M. Verhagen, R. H. H. M. Philipsen, A. Meijers, B. v. Ginneken, "Computer-aided detection of tuberculosis on chest radiographs: An evaluation of the CAD4TB v6 system," Scientific Reports, vol. 10, 2020. Crossref, Google Scholar

[16] S. H. Kassani, P. H. Kassasni, M. J. Wesolowski, K. A. Schneider, and R. Deters, "Automatic detection of coronavirus disease (covid-19) in $\mathrm{X}$-ray and ct images: A machine learning-based approach," arXiv preprint arXiv:2004.10641, 2020

[17] J. P. Cohen, P. Morrison, L. Dao, Covid-19 image data collection, arXiv 2003.11597 (2020). URL: https:/github.com/ieee8023/covidchestxray-dataset.

[18] Adrian Rosebrock, Detecting COVID-19 in X-ray images with Keras, TensorFlow, and Deep Learning, 2020. URL: https://www.pyimagesearch.com/2020/03/16/detecting-covid-19-in-Xray-images-with-keras-tensorflow-and-deep-learning/.

[19] M. Barstugan, U. Ozkaya, S. Ozturk, Coronavirus (COVID-19) classification using ct images by machine learning methods, arXiv preprint arXiv:2003.09424 (2020).

[20] N. Cristianini, J. Shawe-Taylor, et al., An introduction to support vector machines and other kernel-based learning methods, Cambridge university press, 2000.

[21] L.Wang, A.Wong, COVID-net: A tailored deep convolutional neural network design for detection of COVID-19 cases from chest radiography images, arXiv preprint arXiv:2003.09871(2020).

[22] Kaggle, Kaggle's Chest X-Ray Images (Pneumonia) dataset, 2020.URL: https://www.kaggle.com/paultimothymooney/chest-xraypneumonia.

[23] Khan, Asif Iqbal, Junaid Latief Shah, and Mohammad Mudasir Bhat. "CoroNet: A Deep Neural Network for Detection and Diagnosis of COVID-19 from Chest x-Ray Images." Computer Methods and Programs in Biomedicine 196 (2020): 105581. Crossref. Web.

[24]A. Krizhevsky, I. Sutskever, G. E. Hinton, Imagenet classification with deep convolutional neural networks, in Advances in neural information processing systems, 2012, pp. 1097

[25] B. Ghoshal, A. Tucker, Estimating uncertainty, and interpretability in deep learning for coronavirus (COVID-19) detection, arXiv preprint arXiv:2003.10769 (2020).

[26] Chouhan, Vikash, Sanjay Kumar Singh, Aditya Khamparia, Deepak Gupta, Prayag Tiwari, Catarina Moreira, Robertas Damaševičius, and Victor Hugo C. De Albuquerque. "A novel transfer learning based approach for pneumonia detection in chest X-ray images." Applied Sciences 10, no. 2 (2020): 559
[27] S. Rajaraman, J. Siegelman, P. O. Alderson, L. S. Folio, L. R. Folio, and S. K. Antani, "Iteratively pruned deep learning ensembles for covid-19 detection in chest x-rays," arXiv preprint arXiv:2004.08379, 2020

[28] Y.-L. Bureau, J. Ponce, Y. LeCun, A theoretical analysis of feature pooling in visual recognition, in Proceedings of the 27 th international conference on machine learning (ICML-10), 2010, pp. 111 